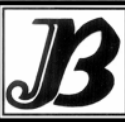

J. bio-sci. 19: 65-75, 2011

ISSN 1023-8654

http://www.banglajol.info/index.php/JBS/index

\title{
NEMATODE DIVERSITY IN BANANA RHIZOSPHERE FROM NORTHERN DISTRICTS OF BANGLADESH
}

\author{
Joynul Abedin, Nuzhat Ara'1, M Sohrab Ali', M Khalequzzaman* \\ Institute of Biological Sciences, University of Rajshahi, Rajshahi, Bangladesh \\ ${ }^{1}$ Department of Zoology, University of Rajshahi, Rajshahi, Bangladesh
}

\begin{abstract}
Context: Banana is one of the most important fruits crops among the different fruit crops in the agricultural and its intensively cultivated in all over the parts of Bangladesh especially in northern part. Many species of plant parasitic nematodes are association with banana root systems and are mainly responsible for controlling the banana productions to greater extend.

Objective: Keeping in view of the disturbing nature of the nematode pest, the present study was undertaken to examine the biodiversity of plant parasitic nematodes associated with banana intensively grown in northern districts of Bangladesh.
\end{abstract}

Materials and Methods: The collection of nematodes was conducted in the banana growing areas of Bogra, Natore, Rajshahi, Rangpur and Gaibandha districts. In each area, banana fields of different commercial cultivars were chosen for collection of soil and root samples. From each field 10 plants were selected randomly for sample collection. Each subsample consisted about $1 \mathrm{~kg}$ of soil and $20 \mathrm{~g}$ of fleshy roots taken from a single mat from a depth of $10-30 \mathrm{~cm}$ and from three equidistant points $(40-50 \mathrm{~cm})$ around the pseudostem Nematodes were extracted from soil by Cobb's sieving technique and from root by Mechanical maceration technique, and processed by Seinhorst's slow dehydration method. Fixing was done in boiling lactophenol cotton blue and finally by formalin glycerin solution. Dehydrated nematodes were kept in glycerol-ethanol solution and mounted permanently on glass slides. Identification was done up to species following the keys.

Results: Among the plant parasitic nematodes ten species belonging to nine genera, six families of a single order, viz. Helicotylenchus dihystera, Rotylenchulus reniformis, Meloidogyne incognita, Hoplolaimus galeatus, Tylenchorhynchus mashhoodi, Tylenchorhynchus annulatus, Criconemoides sp., Hemicriconemoides cocophillus, Cephalenchus emarginatus and Aphelenchus avenae were encountered. Among the non-parasitic nematodes 15 genera belonging to seven families and three orders viz. Acrobeles, Acrobeloides, Aporcelaimus, Aporcelaimium, Aporcelaimellus, Cephalobus, Dorylaimus, Dorylaimoides, Discolaimus, Discolaimium, Discolaimoides, Indodorylaimus, Mylonchulus, lotonchus, and Rhabditis were found to be associated with soils in banana field. It was found that species $\mathrm{H}$. galeatus, $C$. emarginatus and $M$. incognita were present in all the surveyed areas. Four species of parasitic nematodes viz. C. emarginatus, $H$. cocophillus, Criconemoides sp. and $A$. avenae and 5 genera of non parasitic nematodes viz., Aporcelaimium, Aporcelaimellus, Discolaimium, Discolaimoides and Indodorylaimus are reported for the first time in Bangladesh.

Conclusion: Present investigation have clearly indicated that the association of plant parasitic nematodes especially the most an important nematodes species like Hoplolaimus galeatus, Cephalenchus emarginatus and Meloidogyne incognita would cause severe economic yield loss to banana plantation in the study area.

Keywords: Plant parasitic nematode, Free living nematodes, Musa, Nematode diversity.

\section{Introduction}

Banana (Musa $\mathrm{AAA}$ ) and plantain (Musa $\mathrm{AAB}$ ) are among the most important dietary staples in the world (Sharrock and Frison 1999, FAO 2004). In terms of the nutrient contents, banana contains high amounts of carbohydrates, vitamins, fibre and other minerals (Picq and Raymond 1996). In Bangladesh, banana is the top ranking fruits which grows all over the country and available throughout the year. Banana is known to adapt very quickly and produce higher yields under favourable conditions. It is, however, prone to attack by different pathogens like fungi, viruses, bacteria and nematodes. Among the production constraints,

*Corresponding author Email: kzaman@ru.ac.bd 
nematodes constitute one of the major limiting factors to banana production, causing extensive root damage, resulting in serious economic losses (Gold et al. 1994, Barekye et al. 2000). Reports showed that nematodes are a real threat to the banana cultivation (Evaristo 1969, Edwards and Wehunt 1971, Jones and Milne 1982, Sundararaju 2005, Srinivasan et al. 2011). Crop losses caused by nematodes to bananas are very high, with average annual yield losses estimated at about 20\% worldwide (Sasser and Freckman 1987a).

In worldwide basis the most important plant nematode genera are Radopholus, Helicotylenchus, Hemicycliophora, Meloidogyne, Pratylenchus, Paratrichodorus, Scutellonema, Tylenchorhynchus, and Xiphinema (Jones 1979). More than 151 nematode species of 51 genera have been documented worldwide on Musa sp. (Gowen and Quene-herve 1990, Koshy and Banu 2000) and yield losses are estimated to be $19.7 \%$ amounting to US $\$ 17.8$ million (Sasser and Freckman 1987b). Nevertheless, only Radopholus similis, several species of the genus Meloidogyne, Helicotylenchus, Pratylenchus and Rotylenchulus associated with the soil or roots of banana and cause significant losses in banana plantations (Ritzinger et al. 2007). Since no other work has been done on plant parasitic nematodes associated with this ubiquitous and useful fruit crop in northern part of Bangladesh, a survey was made to collect and identify the nematodes associated with banana.

\section{Materials and Methods}

The collection of nematodes was conducted in the banana growing areas of Bogra (Namuja, Mohasthan, Mokamtola), Natore (Tokya, Dhalan, Natore), Rajshahi (Baneshwar, Biraldah, Belpukuria), Rangpur (Mithapukur, Pirgonj, Shatibari) and Gaibandha (Polashbari, Fashitola, Merirhat) districts. Sampling areas were selected where no nematicide was applied in the soil during previous two years. From each field 10 plants were selected randomly for sample collection. Banana plants showing yellow leaves, stunted growth, toppling of the plant and poor growth i.e. diseased plants were preferred.

Each sample consisted ten subsamples, one from each ten randomly selected plants of a plantation. The subsample consisted about $1 \mathrm{~kg}$ of soil and $20 \mathrm{~g}$ of fleshy roots taken from a single mat from a depth of 10$30 \mathrm{~cm}$ and from three equidistant points $(40-50 \mathrm{~cm})$ around the pseudostem (Geetha and Koshy 1984) with the help of hand trowel, spade and knife. All the subsamples of soil and root from each plantation were mixed separately with $5 \mathrm{~kg}$ of soil and $100 \mathrm{~g}$ of roots (composite sample) and were packed in polythene bag (Southey 1970). Composite soil and root samples were collected on the same day of every month at three sites over a period from January 2008 to September 2010.

Nematodes were extracted from soil by Cobb's sieving technique (Cobb 1918) and from root by Mechanical maceration technique (Fallis 1943, Reddy 1983), decanting method followed by modified Baermann's (Baermann 1917) funnel technique (Christie and Perry 1951), processed by Seinhorst's slow dehydration method (Seinhorst 1959). Fixing was done in boiling lactophenol cotton blue (Daykin and Hussey 1985) and finally by formalin-glycerin solution. Dehydrated of nematodes was done in glycerol-ethanol solution. (De Grisse 1969) and mounted permanently on glass slides and identified up to species level following the keys (Thorne 1961, Goodey 1963, Siddiqi 1974, Taylor and Sassor 1978ab). The root-knot nematode species was identified on the basis of the perineal pattern of the mature female.

Measurements were taken according to de Man Formula $(1876,1880)$, modified and amended by Cobb (1914), Thorne (1949) as follows: $n=$ Number of specimens measured; $L=$ Total length of the body; $a=$ Total length divided by the greatest body width; $b=$ Total length divided by the length of esophago-intestinal valve; $\mathrm{C}=$ Total length divided by the tail length; $\mathrm{V}=\%$ of vulval distance ( length from front end to vulva $\times 100) ; c^{\prime}=$ Tail length divided by the tail diameter at anus or cloaca; $S L=$ Stylet length; $S p=$ Spicule length; $R=$ Total number of annules in the body; Ran = Number of annules from anus to tail terminus; RV = Number of annules between tail end and vulva. The above measurements were recorded and mean, SE and range were calculated for confirmation of identification. 


\section{Results}

Two groups of nematodes (plant parasitic and non-parasitic) were found to be associated with banana. Among the plant parasitic nematodes ten species belonging to nine genera, six families of a single order, viz. Helicotylenchus dihystera, Rotylenchulus reniformis, Meloidogyne incognita, Hoplolaimus galeatus, Tylenchorhynchus mashhoodi, Tylenchorhynchus annulatus, Criconemoides sp., Hemicriconemoides cocophillus, Cephalenchus emarginatus and Aphelenchus avenae were encountered. Among the nonparasitic nematodes 15 genera belonging to seven families and three orders viz. Acrobeles, Acrobeloides, Aporcelaimus, Aporcelaimium, Aporcelaimellus, Cephalobus, Dorylaimus, Dorylaimoides, Discolaimus, Discolaimium, Discolaimoides, Indodorylaimus, Mylonchulus, lotonchus, and Rhabditis were found to be associated with soils in banana field. Free living nematodes were identified up to genus depending upon morphology and morphometrics of female specimen.

It was found that species Hoplolaimus galeatus, Cephalenchus emarginatus and Meloidogyne incognita were present in all the surveyed areas (Bogra, Rangpur, Gaibandha, Natore and Rajshahi). Whereas Aphelenchus avenae was found in Bogra, Rangpur and Rajshahi. Hemicriconemoides cocophillus was found in Rajshahi and Bogra. The genus Criconemoides sp. and Tylenchorhynchus annulatus was found in Rajshahi only, Tylenchorhynchus mashhoodi observed in Rangpur, Gaibandha, Natore and Rajshahi. Rotylenchulus reniformis found in Bogra, Natore and Rajshahi. Helicotylenchus dihystera was present abundantly in Bogra, Rangpur and Gaibandha (Table 1). A few adult males and numerous $2^{\text {nd }}$ stage larva of Meloidogyne were present in the soil and the adult females were found within the galled roots of banana.

Four species of parasitic nematodes viz. Cephalenchus emarginatus, Hemicriconemoides cocophillus, Criconemoides sp. and Aphelenchus avenae and 5 genera of non parasitic nematodes viz., Aporcelaimium, Aporcelaimellus, Discolaimium, Discolaimoides and Indodorylaimus were reported for the first time in Bangladesh (Plate 1).

Table 1. Nematodes associated with banana roots and soil.

\begin{tabular}{|c|c|c|c|c|c|}
\hline \multirow{2}{*}{ Nematode } & \multicolumn{5}{|c|}{ Sampling Area } \\
\hline & Borga & Rangpur & Gaibandha & Natore & Rajshahi \\
\hline Aphelenchus avenae & + & + & & & + \\
\hline Cephalenchus emarginatus & + & + & + & + & + \\
\hline Criconemoides sp. & & & & & + \\
\hline Hemicriconemoides cocophillus & + & & & & + \\
\hline Helicotylenchus dihystera & + & + & + & & \\
\hline Hoplolaimus galeatus & + & + & + & + & + \\
\hline Meloidogyne incognita & + & + & + & + & + \\
\hline Rotylenchulus reniformis & + & & & + & + \\
\hline Tylenchorhynchus mashhoodi & & + & + & + & + \\
\hline Tylenchorhynchus annulatus & & & & & + \\
\hline
\end{tabular}

\section{Systematics and Description}

Order: Rhabditida, Family: Rhabditidae

\section{Genus: Rhabditis Dujardin 1845}

Cuticle smooth; 2 part oesophagus with valvulated basal bulb. Stoma tubular,elongate and supported by cuticular 'rhabdions'. Anterior part of oesophagus is cylindrical, the corpus is swollen to form the median bulb which is devoid of valve plates, a narrowing isthmus surrounded by the nerve ring, rounded terminal bulb containing valve plate. Vulva posterior, reproductive system mono-prodelphic, ovary reflexed. Tail elongated and pointed. Female $(n=3): L=610 \pm 15.72(580-632) \mu m, a=18.46 \pm 0.66(17.17-19.33), b=4.6 \pm$ 0.23 (4.14 - 4.88), $c=7.92 \pm 0.68$ (6.6 - 8.86), $V=71.85 \pm 0.88(70.69-73.58) \%$ 
Abedin et al.

Order: Rhabditida, Family: Cephalobidae

\section{Genus: Cephalobus Bastian,1865}

Cuticle annulated, body tapering slightly at extremities, pharyngeal cavity indistinct. Oesophagus long,cylindrical anteriorly,narrowing slightly at isthmus, terminal bulb rounded and valvated. Lip region without probolae, lips duplex. Lateral field reaches to tail tip, Female tail blunt and rounded. Female gonad prodelphic, reflexed down body. Female $(n=3): L=520 \pm 20.82(490-560) \mu \mathrm{m}, a=23.26 \pm 0.6(22.27-$ 24.34), $b=3.2 \pm 0.14(3-3.46), c=16.94 \pm 0.34(16.33-17.5), V=61.02 \pm 1.82(58-64.28) \%$.

Genus: Acrobeles Linstow, 1877

Cuticle annulated, labial probolae long and slender, each bifurcate to about half its length, 6 cephalic probolae about half the length of labial probolae, sharply pointed and each carrying a similar delicate, dentate fringe on either side. Anterior part of oesophagus cylindrical tubular, tapering slightly to short isthmus. Female tail narrowing to pointed. Phasmid at junction of these parts and lateral field ends here. 4 lateral line, two inner lateral line is crenate. Vulva small and pore like. Male tail with 8 pairs of papillae, lateral field runs to tail tip. Female $(n=4): L=504 \pm 12.07(483-524) \mu \mathrm{m}, a=16.24 \pm 0.25(15.75-16.58), b=3.62$ $\pm 0.03(3.58-3.68), c=10.33 \pm 0.19(9.96-10.62), V=57.34 \pm 1.42(55.75-60.17) \%$.

Genus: Acrobeloides (Cobb, 1924) Thorne, 1937

Short stout nematodes with rounded conoid tail. Cuticle annulated, lip region with 3- small, conical, forwardly pointing labial probolae, not furcated. Stoma about a head width long. Cephalic probolae conical like apiculate plates. Lateral field inconspicuous, pre-isthmus portion of oesophagus elongated, fusiform swelling to a spindle-like corpus. Tail conoid and bluntly rounded. Female $(n=3): L=348 \pm 15.03(322-374) \mu \mathrm{m}, \mathrm{a}$ $=16.33 \pm 0.51(15.33-17.0), b=3.63 \pm 0.16(3.34-3.88), c=11.88 \pm 0.30(11.5-12.47), V=66.95 \pm 1.53$ $(64.7-69.87) \%$.

\section{Order: Tylenchida, Family: Criconematidae}

\section{Genus: Criconemoides Taylor, 1936}

Female: Body stout, fusiform, cuticle single layered, thick with large retrose and smooth annules. Head cap or labial disc conspicuous, 1 st annule disc like and projecting forward, spear long, basal knob concave anteriorly appearing hooked. Median oesophageal bulb very large, amalgamated with procorpus. Isthmus short and narrow leading to a slightly swollen cylindrical terminal bulb. Excretory pore opposite to junction of oesophagus and intestine. Anus exceedingly obscure, tail very short, conical. Vulva conspicuous and closed, about $93 \%$, ovary single, prodelphic. Females $(n=3): L=492 \pm 10.1$ (480-512) $\mu \mathrm{m}, \mathrm{a}=20.73 \pm 0.42(20.21-$ 21.56), $b=5.44 \pm 0.13(5.26-5.68), c=19.7 \pm 0.59$ (18.71-20.75), $b^{\prime}=543, c^{\prime}=1.29 \pm 0.005$ (1.28-1.3), $V=$ $93.53 \pm 0.11(93.33-93.66) \%, \mathrm{SL}=50.98 \pm 0.84(49.4-52.25) \mu \mathrm{m}, \mathrm{R}=117.33 \pm 1.45(115-120), \quad \operatorname{Ran}=8.67$ \pm 0.33 (8-9), RV= $10.67 \pm 0.33$ (10-11). Male: not found

Genus: Hemicriconemoides, sp. H. cocophillus Chitwood \& Birchfield, 1957

Female: Body plump, short and slightly curved. Head flat, not offset, with three annules and a head cap or labial disc. $1^{\text {st }}$ annule modified and anteriorly projecting. Procorpus and median oesophageal bulb amalgamated, valves well developed, median bulb very large. Cuticle double, outer cuticle attached to body at head, vulva and tail tip, lacking scales or spines. Annulation well marked and retrose. Isthmus and terminal bulb together forming a short round-ended cylinder. Stylet length about $10 \%$ of body length Spear well developed ,strong and elongated, basal knob large ,upward directed and appearing anchor-shaped.. Vulva posterior, no post vulval sac, ovary mono-prodelphic, out-streched, spermatheca present. Tail short, conical, 9 annules on tail. Anus indistinct on $9^{\text {th }}$ annules from tail terminus. Female $(n=4): L=471 \pm 7.53$ (455.0 487.5) $\mu \mathrm{m}, a=17.88 \pm 0.93(15.31-19.47), b=4.94 \pm 0.1$ (4.68-5.13), $c=18.94 \pm 0.58$ (17.39-20.21), $c^{\prime}=$ $1.26 \pm 0.07(1.14-1.4), V=93.58 \pm 0.07(93.41-93.74) \%, S L=51.77 \pm 1.58(47.5-55.1) \mu \mathrm{m}, \mathrm{R}=122 \pm 0.91$ $(120-124)$, Ran $=8.5 \pm 0.5(8-9), R V=10.5 \pm 0.5(9-11)$. Male: not found 
Order: Tylenchida, Family: Tylenchidae

Genus: Cephalenchus Goodey, 1962 (Geraert, 1968), sp. C. emarginatus

Female: Lip region distinctly offset, stylet weak but with distinct basal knob. Isthmus long and slender, basal bulb pyriform. Body narrowing gradually behind vulva, spermatheca not separated from ovary. Lateral line 6 at middle. Tail long filiform in both sexes. Females $(n=3): L=521 \pm 9.94(501-533) \mu \mathrm{m}, a=32.54 \pm 0.62$ (31.31-33.31), $b=5.93 \pm 0.38$ (5.27- 6.6), $c=3.71 \pm 0.1$ (3.59-3.92), $c^{\prime}=1477 \pm 0.36$ (14.31-15.47), $V=$ $61.11 \pm 1.37(58.71-63.47) \%, S L=15.2 \pm 0.0 \mu \mathrm{m}$. Males $(n=3): L=543 \pm 7.0(532-556) \mu \mathrm{m}, a=31.94 \pm$ 0.41 (31.29 - 32.71), $b=6.45 \pm 0.14$ (6.18 - 6.6), $c=3.01 \pm 0.05$ (2.92 - 3.11), c $=22.82 \pm 0.8$ (21.63 24.34), $S L=15.2 \pm 0.0 \mu \mathrm{m}, \mathrm{Sp}=14.57 \pm 0.32(14.25-15.20) \mu \mathrm{m}$.

Order: Tylenchida, Family: Tylenchorhynchidae

\section{Genus: Tylenchorhynchus Cobb, 1913, sp. T. mashhoodi Siddiqi \& Basir, 1959}

Female: Head slightly offset, hemispherical with 2 annules. Body cylindrical and half C-shaped with thermal death. Head skeleton lightly scelerotized. Spear $17.1 \mu \mathrm{m}$ long with rounded basal knobs. Cuticle prominently annulated, 17annules on tail. Excretory pore level with base of isthmus. Oesophago-intestinal valve hemispherical but inconspicuous. Lateral field with 4 incisures. Phasmids conspicuous about the middle of the tail length. Tail cylindrical with bluntly rounded and broad tip; tail terminus smooth. Vulva median, amphidelphic, both ovaries opposed and outstretched. Male: Tail with similarly placed phasmids. Bursa beginning anterior to spicules and surrounding tail tip. Gubernaculum slightly curved proximally. Females(n=4): $\mathrm{L}=601 \pm 8.32(588-625) \mu \mathrm{m}, \mathrm{a}=31.64 \pm 0.44$ (30.92 - 32.89), $b=4.56 \pm 0.05$ (4.43 - 4.65), $c=13.99 \pm 0.25(13.56-14.69), c^{\prime}=3.02 \pm 0.05(2.87-3.13), V=59.27 \pm 1.09(56.87-61.92) \%, S L=17.1$ $\pm 0.39(16.15-18.05) \mu \mathrm{m}$. Males $(n=3): L=681 \pm 3.61(675-687) \mu \mathrm{m}, a=35.24 \pm 0.45(34.37-35.84), b$ $=4.86 \pm 0.14(4.58-5.0), c=14.74 \pm 0.31$ (14.21 - 15.28), $c^{\prime}=2.64 \pm 0.04(2.57-2.71), S L=17.1 \pm 0.0$ $\mu \mathrm{m}, \mathrm{Sp}=29.67 \pm 0.33(29-30) \mu \mathrm{m}$.

\section{Sp. T. annulatus (Cassidy 1930) Golden 1971}

Female: Head slightly offset, hemispherical with 2 annules. Body cylindrical and C-shaped with thermal death. Head skeleton lightly scelerotized. Spear $19.33 \mu \mathrm{m}$ long with rounded basal knobs. Cuticle prominently annulated. Excretory pore level with base of isthmus. Oesophago-intestinal valve hemispherical but inconspicuous. Phasmids conspicuous about the middle of the tail length. Tail conoid and cylindrical with rounded tip; tail terminus smooth. Vulva median, amphidelphic, both ovaries opposed and outstretched. Female $(n=3): L=610 \pm 39.48(555-687) \mu \mathrm{m}, a=28.13 \pm 0.99(26.43-29.87), b=4.62 \pm 0.2(4.37-$ $5.01), c=14.07 \pm 0.62(13.21-15.27), c^{\prime}=3.27 \pm 0.15(3.0-3.5), S L=19.33 \pm 0.33(19-20) \mu \mathrm{m}, \mathrm{V}=$ $51.79 \pm 0.35(51.24-52.43) \%$.

Order: Tylenchida, Family: Hoplolaimidae

\section{Genus - Hoplolaimus Daday, 1905, sp. H. galeatus (Cobb, 1913) Thorne, 1935}

Female: Lip region rounded, setoff and cap like; cephalic framework massive. Spear $37.0 \mu \mathrm{m}$ long, spear knob massive with anterior projections, tulip shaped. Cuticle coarsely annulated. Dorsal oesophageal gland opening near base of spear knob. Oesophageal glands overlapping intestine mainly dorsally and laterally. Lateral field with 4 lateral lines. Two phasmids located one anterior and the other one posterior to vulva. Tail short rounded, with annules going right round its tip, shorter than width of body at anus. Gonads paired, opposed and outstretched, amphidelphic. Vulva $55 \%$ of body length; spermatheca present, round with full of sperm. Male: Tail surrounded by large bursa extends up to tail tip, spicule paired, stoutly built. Gubernaculum present. Females $(n=5): L=1340 \pm$ $79.57(1170-1637) \mu \mathrm{m}, a=30.31 \pm 2.06(26.0-36.0), b=6.82 \pm 0.29(5.93-7.44), c=44.06 \pm 4.21$ $(36.29-54.58), c^{\prime}=0.86 \pm 0.04(0.75-0.93), V=54.98 \pm 0.74(52.75-56.48) \%, S L=38.47 \pm 0.85(37.05$ 41.8) $\mu \mathrm{m}$. Males $(\mathrm{n}=3)$ : Male- $\mathrm{L}=1172 \pm 75.45(1050-1310) \mu \mathrm{m}, \mathrm{a}=28.53 \pm 1.33(26.25-30.85), b=6.86$ 
$\pm 0.34(6.18-7.27), c=29.94 \pm 1.62(27.65-33.06), c^{\prime}=1.90 \pm 0.08(1.75-2.04), S L=37 \pm 1.15(35-$ 39) $\mu \mathrm{m}, \mathrm{Sp}=42.5 \pm 1.44(40-45) \mu \mathrm{m}$.

Genus: Helicotylenchus Steiner, 1945, sp. H. dihystera (Cobb, 1893) Sher, 1961

Head conical, not offset, moderately sclerotized, 4 annules on head. Cuticle annulated. Body spirally coiled on heat killing (spiral form), DGO almost half of the stylet length. Oesophageal glands overlap the intestine mostly ventrally. Developed stylet with prominent knobs. Basal knobs small and rounded, stylet length $22.61 \mu \mathrm{m}$. Lateral field with 4 incisures. One small phasmid on tail end. Female-Vulva posterior, amphidelphic, tail end concave (more curved dorsally) Phasmids pre-anal, Tail terminus hemispherical to elongated ventrally. Females $(n=5): L=551 \pm 33.57(458-628) \mu \mathrm{m}, a=28.05 \pm 0.79(25.79-30.05), b=$ $4.24 \pm 0.08(3.98-4.45), c=43.35 \pm 2.78(34.44-49.8), c^{\prime}=1.18 \pm 0.06(1.08-1.40), S L=22.61 \pm$ $0.55(20.9-23.75) \mu \mathrm{m}, \mathrm{V}=65.76 \pm 2.81(58.54-71.66) \%$. According to Goody (1963) and C.I.H. set 2 No. 23. Male: not found.

\section{Genus: Rotylenchulus, sp. R. reniformis Linford \& Oliveira, 1940}

Immature females and males vermiform, arcuate on heat killing. Cuticle annulated. Lateral fields with 4 incisures. Cephalic region high, continuous. Stylet 2-3 times long than cephalic region width. DGO about one stylet length behind stylet base. Ovaries paired, with double flexures. Tail elongate-conoid. MalesPhasmids located on tail, spicules slender, lacking distal flanges. Immature females $(n=4): L=375 \pm 7.51$ (362 - 394) $\mu \mathrm{m}, \mathrm{a}=29.85 \pm 0.36(29.31-30.93), b=2.99 \pm 0.13(2.68-3.32), c=14.42 \pm 0.43(13.61-$ 15.47), $c^{\prime}=3.67 \pm 0.06(3.50-3.75), S L=16.39 \pm 0.45(15.2-17.1) \mu \mathrm{m}, V=70.71 \pm 0.3(69.86-71.2) \%$. Male $(n=3): L=411 \pm 8.02(395-420) \mu \mathrm{m}, a=30.08 \pm 0.16(29.86-30.38), b=3.84 \pm 0.03(3.8-3.89), c$ $=18.97 \pm 0.09(18.81-19.10), c^{\prime}=1.81 \pm 0.03(1.75-1.83), S L=14.33 \pm 0.33(14-15) \mu \mathrm{m}, \mathrm{Sp}=21.33 \pm$ $0.33(21-22) \mu \mathrm{m}$.

Order: Tylenchida, Family: Meloidogynidae

\section{Genus: Meloidogyne Goeldi, 1887, sp. M. incognita (Kofold \& White, 1919) Chitwood, 1949}

Female: Body swollen to spherical with short projecting neck. Cap like structure on head. Head with 2 annules behind head cap. Spear knobs rounded and drawn out laterally. Vulva and anus close together, terminal. Ovaries prodelphic, convoluted. Male: Males elongate, cylindrical, vermiform. Head skeleton well developed, head not offset, annulated. Cuticle annulated, spear well developed with rounded basal knobs. Lateral field with 4 incisures of which inner two is smooth and the outer two crenate. Tail very short, bluntly rounded, hemispherical, without bursa. Slightly curved spicules, gubernaculum present. Perineal pattern: Dorsal arch high, rounded, striae closely spaced, very wavy to zig-zag especially dorsally and laterally. Perineum with fingerprint-like cuticular pattern. Vulva and anus close together, terminal. Males $(n=3): L=$ $1581 \pm 164.58(1300-1870) \mu \mathrm{m}, a=41.62 \pm 3.91$ (34.67 - 48.19), $b=7.83 \pm 1.13(6.12-9.97), c=160.57$ $\pm 9.2(150.72-178.95), c^{\prime}=0.69 \pm 0.03(0.65-0.75), S L=18.43 \pm 0.9(17.1-20.15) \mu \mathrm{m}, \mathrm{Sp}=28.38 \pm$ $2.32(23.75-31) \mu \mathrm{m}$.

Order: Tylenchida, Family: Aphelenchidae

\section{Genus: Aphelenchus sp. A. avenae Bastian, 1865}

Head not offset, body tapering anteriorly. Stylet short with basal thickening, spear knob absent. Mucro absent. Lateral fields with about 10 incisures. Vulva posterior about $75 \%$, posterior uterine sac reduced, prodelphic, ovary outstretched. Tail cylindrical, tail tip bluntly rounded. Median bulb large and placed anteriorly, occupied almost whole body width long oesophageal gland overlap dorsally. Females $(n=4): L=$ $580 \pm 38.68$ (515 - 689) $\mu \mathrm{m}, \mathrm{a}=39.97 \pm 1.37$ (36.14 - 42.66), $b=3.88 \pm 0.24(3.43-4.53), c=25.08 \pm$ $0.57(23.4-25.81), c^{\prime}=3.36 \pm 0.19(3.0-3.87), V=74.92 \pm 0.54(73.37-75.76) \%, S L=13.3 \pm 0.39$ $(12.35-14.25) \mu \mathrm{m}$. 
Order: Mononchida, Family: Mononchidae

Genus: Mylonchulus ( Cobb,1916) Pennak, 1953

Head not distinctly offset, continuous with body. Cuticle smooth. Stoma strongly cuticularized, cavity like, wide and oblong. Dorsal tooth in the posterior half of the stoma, pointing forwards, large, opposed by 3 subventral teeth all pointing forward. Oesophagus stout, muscular, glandular and cylindrical. Tail long with caudal glands. Female $(n=3): L=1070 \pm 26.46(1030-1120) \mu \mathrm{m}, a=21.79 \pm 0.94(20.36-23.55), b=3.83$ \pm 0.09 (3.73 - 4.0), $\mathrm{C}=27.28 \pm 1.11(25.75-29.44), \mathrm{V}=57.11 \pm 1.38(54.36-58.49) \%$.

\section{Genus: lotonchus}

Male : Head not offset, continuous with body. Cuticle smooth. Stoma strongly cuticularized, cavity like, wide and oblong, barrel shaped and flat at base. Dorsal tooth in the posterior half of the stoma, pointing forwards, large, opposed by 3 subventral teeth (denticles) all pointing forward. Oesophagus stout, muscular, glandular and cylindrical. Tail long filiform with caudal glands. Female not found. Male $(n=3): L=1438 \pm 70.49$ (1300 1532) $\mu \mathrm{m}, a=28.03 \pm 1.52(25.0-29.64), b=3.83 \pm 0.03(3.77-3.87), c=6.1 \pm 0.31(5.63-6.68), S p=$ $46.67 \pm 0.83(45-47.5)$.

Order: Dorylaimida, Family: Dorylaimidae

Genus: Dorylaimus Dujardin,1845

Cuticle smooth and thick with distinct longitudinal ridges, body tapering at both ends. Lip region continuous with body contur. Odontostyle strong and well developed, guiding ring double, odontophore rod-like. Amphid with stirrup-shaped fovea, cardia conoid to elongate. Expanded part of oesophagus about one half of oesophageal length. Female gonad paired, amphidelphic, opposed and reflexed. Testes paired, opposed, outstretched. Tail long filiform in female and short conoid with rounded terminus in males. Female $(n=3): L=$ $3413 \pm 141.11$ (3200-3680), $\quad a=32.44 \pm 0.95(30.55-33.45), b=4.92 \pm 0.1(4.8-5.11), \quad c=11.37 \pm 0.26$ (11.03-11.87), V= $41.43 \pm 0.88(40.47-43.2) \%$.

Genus: Indodorylaimus Ali and Prabha, 1974

Lip region with sclerotized framework, truncated and distinctly narrower than adjoining body. Odontostyle cylindrical, odontophore rod-like. Guiding ring single. Basal expanded part of oesophagus half of total oesophageal length. Female reproductive system mono-opisthodelphic, anterior genital branch reduced to a uterine sac. Males with dorylaimoid spicules. Tail long filiform, similar in sexes. Female( $n=3): L=1410 \pm$ 15.56 (1387 - 1440) $\mu \mathrm{m}, a=43.33 \pm 1.77$ (41.14 - 46.83), $b=5.0 \pm 0.07$ (4.87 - 5.11), $c=6.03 \pm 0.14$ (5.76 - 6.24), $V=30.7 \pm 0.32(30.21-31.29) \%$.

Order: Dorylaimida, Family: Discolaimidae

\section{Genus: Discolaimus Cobb, 1913}

Cuticle smooth, lateral chord with glandular bodies. Lip region broadly discoid, disc-like. Odontostyle long, narrow; odontophore rod-like. Oesophagus 2 parts- a narrower anterior region about one-third its length and a posterior cylindrical expanded part about two-third of its length. Cardia disc like. Female reproductive system amphidelphic. Tail short conoid and bluntly rounded. Female $(n=3): L=1151 \pm 28.35$ (1095 - 1185) $\mu \mathrm{m}, \mathrm{a}=28.55 \pm 0.4(27.76-29.0), b=4.05 \pm 0.08(3.95-4.2), c=39.15 \pm 0.49(38.23-39.88), V=49.99 \pm$ $1.14(48.1-52.05) \%$

\section{Genus: Discolaimoides Heyns, 1963}

Body slender, tapering anteriorly. Cuticle smooth. Lip region offset by an expansion, not offset by constriction, not discoid. Lateral glands conspicuous. Odontostyle short, odontophore rod-like. Vagina not sclerotized. Female reproductive system amphidelphic. Tail slightly elongated and conoid, similar in sexes. Female $(n=3): L=1651 \pm 34.44$ (1610 - 1720) $\mu \mathrm{m}, a=44.23 \pm 0.53$ (43.51 - 45.26), $b=4.6 \pm 0.1$ (4.45 $4.78), c=35.52 \pm 1.29(33.05-37.39), V=44.8 \pm 0.48(43.89-45.54) \%$ 

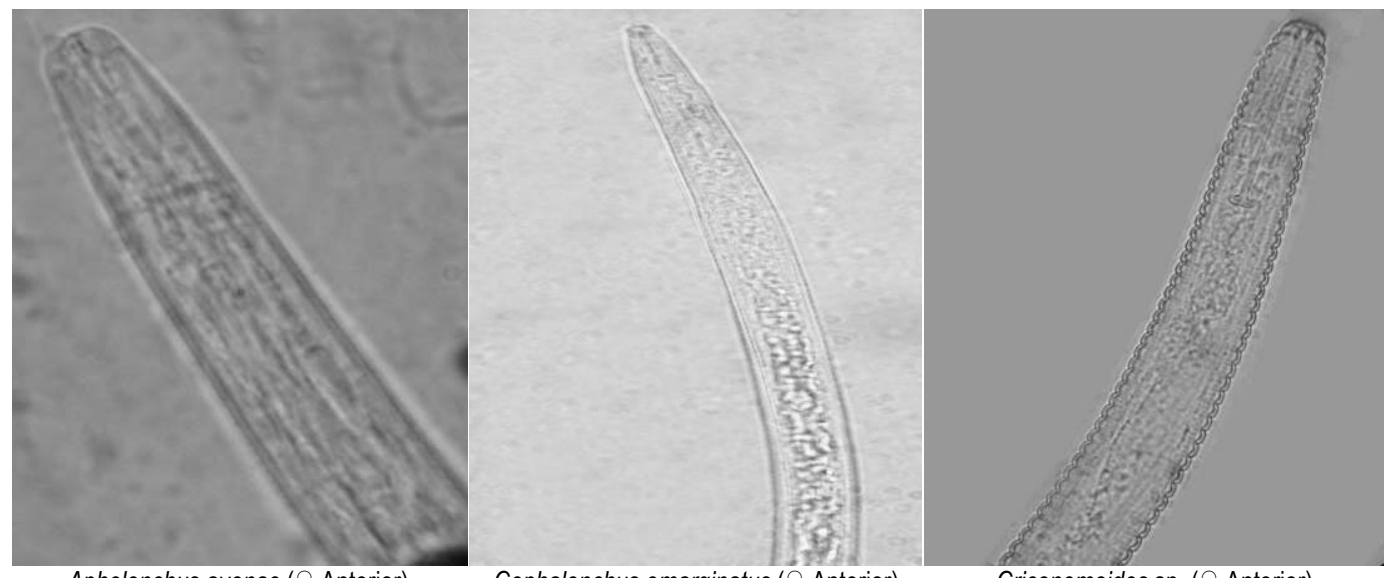

Aphelenchus avenae (q Anterior)

Cephalenchus emarginatus ( 9 Anterior)

Criconemoides sp. ( $Q$ Anterior)
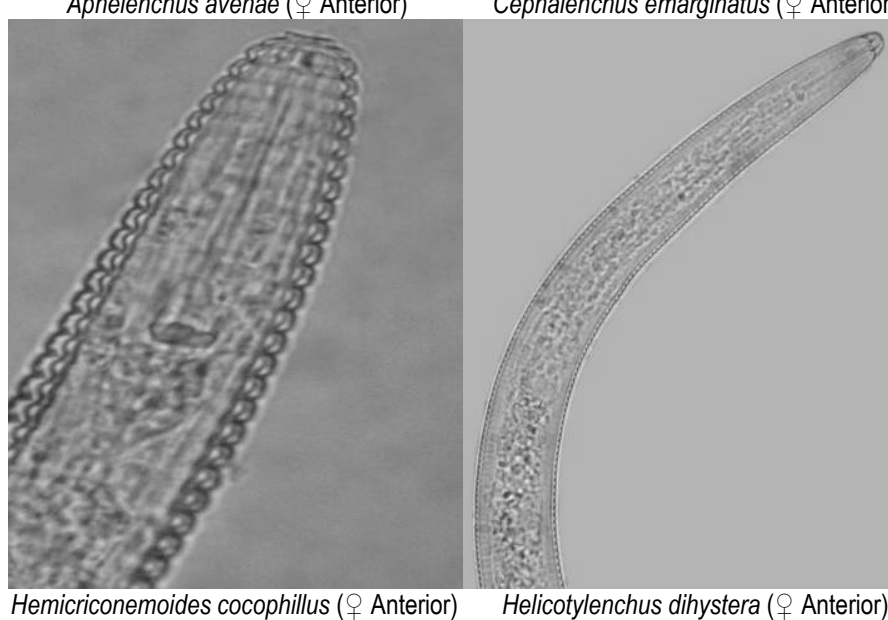

Helicotylenchus dihystera ( 9 Anterior)

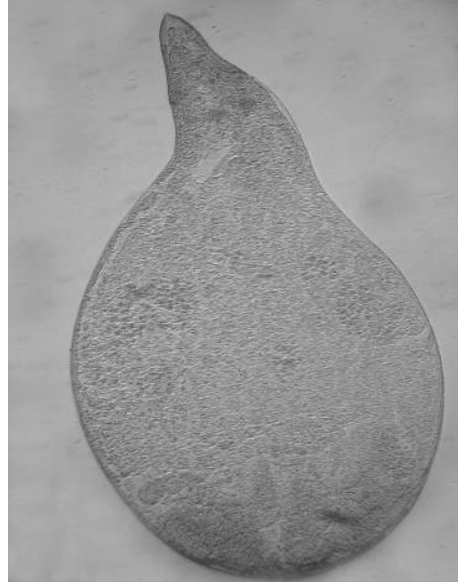

Meloidogyne incognita (ㅇ entire)

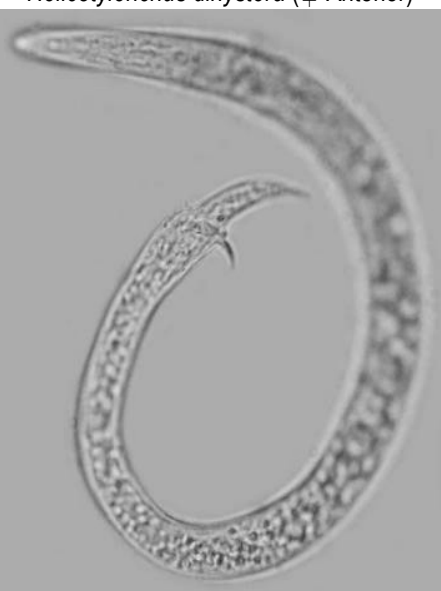

Rotylenchulus reniformis ( 0 entire)

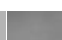

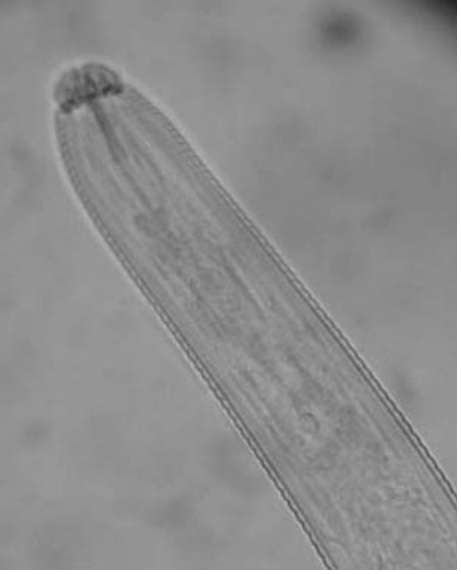

Hoplolaimus galeatus (+ Anterior)

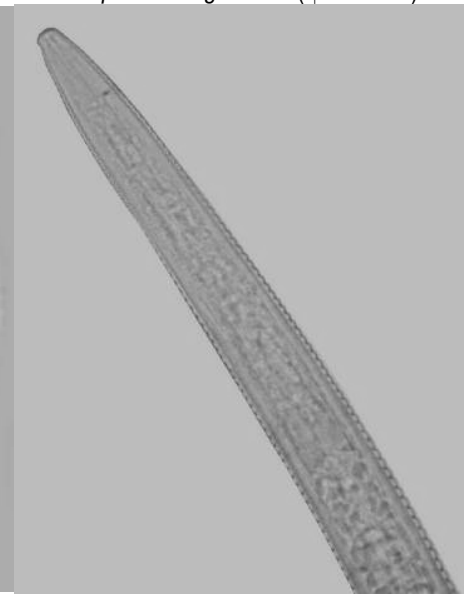

Tylenchorhynchus mashhoodi ( + Anterior)

Plate 1. Microphotographs of the banana associated nematodes 


\section{Genus: Discolaimium Thorne, 1939}

Body slender, tapering anteriorly. Cuticle smooth. Lip region offset by deep constriction, discoid but same wide as body. Lateral glands conspicuous. Odontostyle short dorylaimoid. Guiding ring single. Anterior part of oesophagus slender, without musculature and expanded basal part. Sheath-like membrane sometimes encircles base of expanded part of oesophagus. Vagina not sclerotized. Female reproductive system amphidelphic. Tail slightly elongated and conoid, similar in sexes. Female( $n=3): L=1210 \pm 55.08(1100$ 1270) $\mu \mathrm{m}, \mathrm{a}=33.6 \pm 0.36$ (33.16-34.32), $b=3.63 \pm 0.16$ (3.45-3.94), $c=42.08 \pm 1.2(39.69-43.45), V=$ $50.07 \pm 0.54(49.05-50.91) \%$

Order: Dorylaimida, Family: Aporcelaimidae

\section{Genus: Aporcelaimus Thorne \& Swanger, 1936}

Cuticle smooth and thick with criss-cross lines. Body strongly tapering towards anterior end. Lip region offset by deep constriction, lips distinctly separate. Odontostyle as long as lip region width. Oesophagus expanding gradually near middle, posterior part of oesophagus massive. Cardiac disc present. Female reproductive system amphidelphic, gonads paired, opposed, reflexed. Vulva with sclerotized labia. Tails short, conoid. Males with dorylaimoid spicules. Female $(n=3): L=2183 \pm 123.5(1985-2410) \mu \mathrm{m}, a=30.72 \pm 2.15(26.97-$ 34.42), $b=3.64 \pm 0.11$ (3.45-3.82), $c=63.08 \pm 2.99(59.93-69.07), V=53.7 \pm 1.4(51-55.68) \%$

\section{Genus: Aporcelaimium Loof and Coomans, 1970}

Cuticle smooth and thick without criss-cross lines or punctuation. Lips conspicuous, lip region setoff by deep constriction, lip region narrow. Odontostyle dorylaimoid, with wide aperture, odontophore simple rodlike. Oesophagus expanding gradually near middle. Cardia developed, oesophago-intestinal disc absent. Female reproductive system amphidelphic, ovaries short, reflexed to near the vulva.Vulva without sclerotization. Tails short, conoid. Males with dorylaimoid spicules. Female( $n=2): L=2098 \pm 28$ (20702126) $\mu \mathrm{m}, \mathrm{a}=37.65 \pm 0.87$ (36.78-38.51), $\mathrm{b}=4.25 \pm 0.005$ (4.24-4.25), $\mathrm{c}=36.11 \pm 0.68(35.43-36.78), \mathrm{V}=$ $54.73 \pm 0.54(54.19-55.27) \%$

\section{Genus: Aporcelaimellus Heyns,1965}

Robust body. Cuticle thickened towards body end. Lip region setoff by deep constriction, lips angular, separated. Odontostyle dorylaimoid. Cardiac disc thin. Female reproductive system amphidelphic, ovaries two, vulva transverse, without labial sclerotization. Tail short, bluntly rounded. Female $(n=3): L=1514 \pm$ $23.44(1470-1550) \mu \mathrm{m}, \mathrm{a}=23.96 \pm 0.96(22.62-25.83), b=4.34 \pm 0.22(4.08-4.78), c=49.89 \pm 2.87$ (44.55-54.38), $V=54.4 \pm 0.64(53.23-55.44) \%$.

Order: Dorylaimida, Family: Mydonomidae

Genus: Dorylaimoides Thorne and Swanger, 1936

Female: Cuticle and sub-cuticle finely striated. Lip region slightly offset odontostyle asymmetrical, dorsal arm longer than ventral. Guiding ring single. Odontophore arcuate. Oesophageal bulb cylindroid. Expanded part of oesophagus is half of the total oesophageal length. Female reproductive system amphidelphic. Tail short ,conoid to hemispheroid, similar in sexes. Female $(n=3): L=665 \pm 8.66(650-680) \mu \mathrm{m}, a=22.94 \pm 0.16$ (22.67-23.21), b = $3.55 \pm 0.02$ (3.51-3.58), $c=32.44 \pm 0.03$ (32.38-32.5), $V=60.19 \pm 0.56(59.23-61.18) \%$.

\section{Discussion}

There are four species of nematodes reported in the banana fields in Bangladesh, i.e. burrowing, spiral, root-knot and lesion nematodes. A survey was conducted by Chowdhury et al. (1981) at Joydebpur to identify nematode genera associated with banana. They collected root and soil samples from several banana plantations and identified six nematode genera. They were Pratylenchus spp., Helicotylenchus spp., Hoplolaimus spp., Hirschmanniella spp., Tylenchorhynchus spp. and Meloidogyne sp., which were found to be directly associated with root damage. Another survey was conducted by Mian (1986) throughout Bangladesh to record the plant- 
parasitic nematodes associated with some important crop species commonly grown in the country. Among them, Helicotylenchus multicinctus, Hoplolaimus sp., Meloidogyne incognita, M. javanica, Radopholus similis, Tylenchorhynchus claytoni were associated with banana.

Seventyone nematode species of 33 genera are known to be associated with banana (Krishnappa and Reddy 1995, Koshy and Sosamma 2001) in India. Only seventeen species of plant parasite nematodes have been recorded from West Bengal (Mukherjee and Dasgupta 1983) and Pratylenchus, Meloidogyne, Helicotylenchus, Tylenchorhynchus, Hoplolaimus, Rotylenchulus, Hirschmanniella, and Criconemoides are common genus in banana (Khan and Hasan 2010). Srinivasan et al. (2011) recorded P. coffeae, $M$. incognita, R. simillis and $H$. multicintus as major and Hetroderaoruzicola sp., Tylenchorhynchus sp., Paratylenchus sp., Rotylenchus sp., Longiderus sp., and Peralongidorus sp. as minor pest nematode of banana in Tamilnadu, India.

\section{Conclusion}

Present investigation have clearly indicated that the association of plant parasitic nematodes especially the most an important nematodes species like Hoplolaimus galeatus, Cephalenchus emarginatus and Meloidogyne incognita would cause severe economic yield loss to banana plantation in the study area, if the management practices are not being done to kept the nematodes population under check. In order overcome these problems, integrated nematode management schedule be adopted at the earliest for greater yield of bananas in those areas.

\section{Acknowledgements}

This work is a part of the PhD thesis of the first author, he is thankful to the Institute of Biological Sciences, University of Rajshahi, for offering his fellowship. We are thankful to the IBSc and Department of Zoology, University of Rajshahi, for providing all the necessary laboratory facilities during this work.

\section{References}

Baermann G. 1917. Eine einfache Methode zur Auffindung yon Ankylostomum (Nematoden) larven in Erdproben. Geneesk Tijdschr Ned-Indie 57, 131-137

Barekye A, Kashaiga IN, Tushemereirwe WK, Adipala E. 2000. Comparison of damage levels caused by Radopholus similis and Helicotylenchus multicinctus on bananas in Ugands. Ann Appl Biol 137, 273-278. http://dx.doi.org/10.1111/j.17447348.2000.tb00068.x.

Christie JR, Perry VG. 1951. Removing nematodes from soil. Proc Helminthol Soc Washington 18, 106 -108.

Cobb NA. 1914. Antarctic marine free-living nematodes of the Shakelton expedition. Contributions to a Science of Nematology 3-33.

Cobb NA. 1918. Estimating the Nema Population of the Soil. Agricultural Technology Circular I. Bureau of Plant Industry, Department of Agriculture, United States.

Daykin ME, Hussey RS. 1985. Staining and histopathological techniques in nematology. In: An advances treatise on Meloidogyne spp. Vol. II. N. C. State Univ. NC,. USA. pp. 39-48.

DeGrisse AT. 1969. Redescription ou modifications de quelques techniques utilisées dans l'étude des nematodes phytoparasitaires. Meded. Rijksfakulteit Landbouwwetens chappen Gent 34, 351-369.

De Man JG. 1876. Onderzoekingen over vrij in de aarde levende nematoden. Tijdschr Ned. Dierk. Ver 2, 78-196.

De Man JG. 1880. Die Eingheimischen, frei in der reinen erde und im süssen wasser lebends Nematoden. Tijdschr Ned Dierk Ver 5, 1-104

Edwards DI, Wehunt EJ. 1971. Host range of Radopholus similis from banana areas of Central America with indication of additional races. Plant Dis Rep 55, 415-418.

Evaristo NF. 1969. Contribuçao para o reconhecimento nematologico das bananeiras em moçambique. Agronomia Moçambicana 3 , 169-178

Fallis AM. 1943. Use of the Waring blendor to separate small parasites from tissue. Can T Publ Hlth 34, 44.

FAO, 2004. FAO Statistical Database. Available at www.faostat.fao.org.

Geetha SM, Koshy PK. 1994. Standardisation of sampling zones for detection and survey of Radopholus similis in the rhizosphere of various crops. Indian J Nematol 24, 120-124. 
Gold CS, Speijer PR, Karamura EB, Tushemereirwe WK, Kashaija IN. 1994. Survey methodologies for banana weevil and nematode damage assessment in Uganda. Afr Crop Sci J 2, 309-321.

Goodey T. 1963. Soil and fresh water nematodes. Methuen \& Ltd. London.

Gowen SR, Queneherve P. 1990. Nematode parasites of bananas, plantains and abaca. In: Plant Parasitic Nematodes in Subtropical and Tropical Agriculture (Luc M, Sikora RA, Bridge J, eds.), CABI Publishing, Wallingford, UK. pp. 431-460.

Jones RK. 1979. Nematodes associated with bananas in South Africa. Phytophylactica 11, 79-81.

Jones RK, Milne DL. 1982. Nematode pests of banana. In: Nematology in South Africa, 30-37pp. Keetch DP, Heyns J. (eds). Dept Agric Fisheries, Rep South Africa, Sci. Bull. No. 400.

Khan MR, Hasan MA. 2010. Nematode diversity in banana rhizosphere from West Bengal, India. J Pla Proc Res 50, 263-268. http://dx.doi.org/10.2478/v10045-010-0046-9.

Koshy PK, Banu BJ. 2000. Nematode diversity in plantation crops: the world scenario. 5. In: Nematode Diversity (Jairajpuri MS ed.) p. 390-402. Maulana Azad National Urdu University, Hyderabad, India, 500pp.

Koshy PK, Sosamma VK. 2001. Nematode diseases of plantation crops and their management. National Congress on Centenary of Nematology in India- Appraisal and Future Plans, Dec.5-7, 2001, Division of Nematology, Indian Agricultural Research Institute, New Delhi, India, pp. 48-49.

Krishnappa K, Reddy BMR. 1995. Nematode problems of banana in India. In: Nematode pest management - an appraisal of ecofriendly approaches (Swarup G, Dasgupta DR, Gill JS, eds.) pp.233-238, Nematological society of India, New Delhi, India,.

Mian IH. 1986. Plant parasitic nematodes associated with some crop species in Bangladesh. Bangladesh. J. Plant Pathol 2 (1), 7-13.

Mukherjee B, Dasgupta MK. 1983. Commonity analysis of nematodes associated with banana populations in the Hooghly district, West Bengal. Nematol medit 11(1), 43-48.

Picq C, Raymond R. 1996. Making the most of Musa. INIBAP. Montpellier, France. 15pp.

Reddy PP. 1983. Plant Nematology. Agricole Publishing Academy, New Delhi, India, 287pp.

Ritzinger CHSP, Borges AL, Ledo CA, Caldas RC. 2007. Plant parasitic nematodes associated with banana 'Pacovan' in irrigated condition: connections with production. Revista Brasileira de Fruticultura 29(3), 677-680. http://dx.doi.org/10.1590/S010029452007000300048

Sasser JN, Freckman DW. 1987a. A world perspective on Nematology: the role of the society. In: Vistas on Nematology p.28-34. (Veech JA, Dickson DW, eds.). Hyattville, MD, Society of Nematologists, 509 pp.

Sasser JN, Freckman DW. 1987b. A world perspective on Nematology: the role of resistance of banana cultivars to the nematodes Radopholus similis (Cobb) Thorne and Meloidogyne incognita Chitwood. Phil Agriculturist 68, 335-349.

Seinhorst JW. 1959. A rapid method for the transfer of nematodes from fixative to anhydrous glycerine. Nematologica 4, 67- 69. http://dx.doi.org/10.1163/187529259X00381.

Sharrock S, Frison E. 1999. Musa production around the world-trends, varieties and regional importance. In: Networking banana and plantain: INIBAP Annual Report 1998. INIBAP, Montpellier, France, pp. $42-47$.

Siddiqi MR. 1974. Tylenchulus semipenetrans. C.I.H. Descriptions of Plant-parasitic Nematodes. Set 3, No. 34. Commonwealth Institute of Parasitology. C.A.B. International, 4.

Southey JF. 1970. Laboratory Methods for Works with Plant and Soil Nematodes. Tech Bull Min Agric Fish, 2, London (5 ${ }^{\text {th }}$ ed), 148pp.

Srinivasan R, Kulothungan S, Sundararaju P, Govindasamy C. 2011. Biodiversity of plant parasitic nematodes associated with banana in Thanjavur District of Tamil Nadu. Int J Plant Anim Env Sci 1(2), 63-69.

Sundararaju P. 2005. Biodiversity of plant parasitic nematodes associated with banana in northern parts of Tamil Nadu, India. Current Nematol 18 (1\&2), 27-33.

Taylor AL, Sasser JN. 1978a. Biology, Identification and Control of Root-Knot Nematodes. Raleigh, NC, USA, North Carolina State University Graphics, $111 \mathrm{pp}$.

Taylor AL, Sasser JN. 1978b. International Meloidogyne Project, contract No. AID/ta-c-1234. Biology, Identification and Control of Root Knot Nematode (Meloidogyne spp).

Thorne G. 1949. On the classification of the Tylenchida, new order (Nematode, Phasmidia). Proc Helmithol Soc Washington 16, 37-73.

Thorne G. 1961. Principles of Nematology. McGraw-Hill Book Company, INC. New York, Toronto, London, 553pp. 